\title{
Cicatriz hipertrófica posqueiloplastía de fisura labial: Revisión del tratamiento actual
}

\author{
Hypertrophic scar after cleft lip repair: Review of the current treatment
}

\begin{abstract}
Alberto Córdova-Aguilar 1,a,b,c
RESUMEN

La fisura labial -con o sin fisura palatina- es la malformación congénita facial más frecuente a nivel mundial que requiere cirugía -queiloplastía- para ser corregida. Desafortunadamente, la cicatriz hipertrófica posqueiloplastía es un resultado común que afecta negativamente al paciente, su entorno social e incluso a los especialistas, pues a la fecha no existe tratamiento específico. Con el objetivo de conocer el estado actual del tratamiento de dicho problema, se realizó una revisión bibliográfica desde el año 2000 en las diferentes bases de datos. Se encontró que las opciones de tratamiento y prevención son similares: el cierre primario de la herida sin tensión, la cinta adhesiva de papel microporoso, la inyección de corticoide intralesional, la lámina o gel de silicona, el gel de flavonoides, y el láser. Se concluye que el tratamiento de la cicatriz hipertrófica posqueiloplastía aún es insatisfactorio y la investigación limitada al no ser una patología replicable en modelos animales.
\end{abstract}

PALABRAS CLAVE: Labio leporino, cicatrización de heridas, cicatriz hipertrófica. (Fuente: DeCS BIREME).

\section{SUMMARY}

Cleft lip -with or without cleft palate- is the most frequently reported facial congenital malformation in the world that requires surgery -cheiloplasty- to be corrected. Unfortunately, the hypertrophic scar after cheiloplasty is a common result that negatively affects the patient, their social environment and even the specialists, because to date there is no specific treatment. In order to know the current status of the treatment of this problem, a literature review was conducted since 2000 in the different databases. It was found that the treatment and prevention options are similar: primary closure of the wound without tension, adhesive tape of microporous paper, injection of intralesional corticoid, silicone sheet or gel, flavonoid gel, and laser. It was concluded that the treatment of the hypertrophic scar after cheiloplasty is still unsatisfactory and the research is limited since it is not a replicable pathology in animal models.

KEYWORDS: Cleft lip, wound healing, cicatrix, hypertrophic. (Source: MeSH NLM).

\section{INTRODUCCIÓN}

La fisura labial -con o sin fisura palatina- es un problema de salud pública mundial (1). Los pacientes que padecen esta malformación congénita facial requieren al menos de una intervención quirúrgica al labio -queiloplastía- a partir de los tres meses de edad para corregir este defecto. Pese a recibir el tratamiento quirúrgico oportuno, hasta $36,3 \%$ de estos pacientes desarrollan entre tres a seis meses posqueiloplastía una cicatriz hipertrófica en el labio superior, siendo más propensos los asiático-orientales e hispanos (2).

\footnotetext{
Universidad Nacional Mayor de San Marcos, Lima, Perú.

Médico especialista en Cirugía Plástica.

Magíster en Salud Pública.

Docente universitario.
} 
En la cicatriz normal posqueiloplastía, la dermis afectada es reemplazada por un tejido fibroso escaso de forma asintomática; a diferencia de la cicatriz hipertrófica que presenta abundante tejido fibroso y síntomas locales como el dolor, el eritema y el prurito (3). Naturalmente una cicatriz hipertrófica posqueiloplastía es difícil de ocultar pues genera un estigma facial que afecta psicológica y emocionalmente a quienes la padecen. Al mismo tiempo genera un verdadero desafío para los especialistas, ya que a la fecha no existe un tratamiento específico para este tipo de cicatriz (4).

Esta revisión se realizó con el objetivo de conocer el estado actual del tratamiento de la cicatriz hipertrófica posqueiloplastía. Para tal fin, se realizó una búsqueda de la literatura en las bases de datos PUBMED, LILACS, MEDLINE, PLoS, SciELO, Redalyc y encontrados por Google Académico; se seleccionaron trabajos disponibles en texto completo desde el año 2000 en idioma español e inglés; se utilizaron las palabras clave cicatrización, tratamiento cicatriz hipertrófica, labio leporino, fisura labial, queiloplastía.

\section{Enfoque terapéutico}

Existen varios mecanismos para restaurar la función tisular luego de una lesión que van desde la regeneración completa del tejido -como en los fetos de humanos a mediados de la gestación- hasta la formación de una cicatriz en los organismos posnatales (5). Ahora bien, en los pacientes operados el proceso de cicatrización culmina con la reparación fibrótica del tejido afectado. Cuando esta reparación es excesiva puede originar dos tipos de cicatrización, la hipertrófica o el queloide (6). Ambos tipos cicatrización son consideradas patológicas y por razones aún desconocidas, son enfermedades exclusivas del ser humano (7). Ciertamente, la cicatriz hipertrófica es la forma más común y difiere del queloide en la preservación de la piel sana circundante al tejido cicatrizal (8).
Pese a que el mecanismo exacto de la cicatrización es desconocido (9), se sabe que la formación de una cicatriz normal comprende un proceso de maduración que comúnmente dura 12 meses y se divide en tres fases, la inflamatoria, la proliferativa y la remodelación, que involucra tanto la síntesis como la degradación del tejido para evitar la formación excesiva de fibrosis (10). Cualquier alteración en este proceso puede determinar la formación de una cicatriz patológica.

Existen diversos factores que predisponen a la producción de una cicatriz hipertrófica como: la etnicidad, la ubicación anatómica de la incisión, la técnica quirúrgica y las infecciones posoperatorias (11). En este sentido, la piel del labio superior tiene un riesgo elevado de hacer una cicatriz hipertrófica por los movimientos repetitivos del músculo orbicular de los labios al hablar, comer, beber, etc. y con la expresión facial que transmite fuerzas de tensión que distraen y ensanchan la herida (12).

\section{PLAN DE TRATAMIENTO}

Todo plan de tratamiento comienza con el correcto diagnóstico. Para ello, es de vital importancia diferenciar entre la cicatriz madura, la inmadura y la hipertrófica (tabla 1). Una cicatriz madura es el resultado normal de la reparación de tejidos, es frágil, inelástica y carece de apéndices cutáneos (folículos pilosos y glándulas sudoríparas) (13).

\section{Tratamiento conservador}

Ante una cicatriz hipertrófica posqueiloplastía, el tratamiento conservador suele ser la primera opción para solucionar el problema. La estrategia actual tanto para el tratamiento como para la prevención es similar; se basa en disminuir la inflamación (4), así como evitar la mayoría de los factores predisponentes. Existen diversas terapias, pero solo algunas precisan la tasa de éxito o de no recurrencia, por lo que muchas veces la decisión se basa en la experiencia clínica del especialista:

Tabla 1. Tratamiento de la cicatriz posqueiloplastía.

\begin{tabular}{lll}
\hline Cicatriz & Características & Tratamiento \\
\hline Madura normal & Hipo o hiperpigmentada, plana & Ninguno \\
Inmadura & Roja, ligeramente elevada, a veces dolorosa & Conservador / Preventivo \\
& & $1^{\circ}$ línea: Lámina o gel de silicona \\
Hipertrófica & Elevada, pica, duele, confinada a bordes & $2^{\circ}$ línea: Corticoide intralesional \\
& & $3^{\circ}$ línea: Revisión quirúrgica \\
\hline
\end{tabular}




\section{a) Cinta adhesiva de papel microporoso}

Es la alternativa más económica y de fácil acceso. $\mathrm{Su}$ uso se fundamenta en el bloqueo de la mecanotransducción. Este último, es un proceso por el cual la fuerza mecánica, es decir, la tensión ejercida sobre la piel de una herida genera múltiples respuestas bioquímicas inflamatorias que predisponen a una mala cicatriz (14). Esta cinta adhesiva de papel microporoso además de reducir la tensión de la herida, evita la deshidratación (15).

\section{b) Lámina o gel de silicona}

Estos productos además de inhibir la mecanotransducción han demostrado acelerar la maduración de la cicatriz hipertrófica y disminuir los síntomas asociados -dolor y prurito- (16,17). Se aplican directamente sobre la cicatriz y son superiores a las cintas adhesivas de papel microporoso porque evitan la avulsión epidérmica repetida (4). Otro mecanismo de acción de la lámina de silicona es la presión e hidratación de la superficie de la cicatriz (18). En cualquiera de sus dos presentaciones, se recomienda su uso a partir de la tercera semana poscirugía por más de 12 horas al día durante al menos 2 meses (4). Una ventaja adicional del gel de silicona es que puede usarse en áreas del cuerpo donde la lámina no encaja fácilmente o en pacientes que no colaboren (19). Sin embargo, al comparar la eficacia de ambas formas no se observaron diferencias (20). El efecto adverso más común es la irritación local de los tejidos que muchas veces requiere la suspensión del uso.

\section{c) Corticoesteroide intralesional}

El uso de corticoide intralesional ofrece una segunda línea de terapia para las cicatrices hipertróficas refractarias a la lámina o gel de silicona (21), pero debe usarse con precaución. El corticoide se inyecta en la dermis de la cicatriz. Esta aplicación, intralesional o intradérmica, es el método más usado para reducir rápidamente el volumen de la cicatriz hipertrófica; sin embargo, las cintas con emplastos de esteroides y las cremas de esteroides también son buenas opciones en pacientes pediátricos y cuando están disponibles en el mercado (22).

El mecanismo de acción exacto se desconoce, pero se atribuye a su efecto antiinflamatorio tras inducir la vasoconstricción local y finalmente la reducción en la síntesis de colágeno (18). El corticoide intralesional más usado es la Triamcinolona a una dosis variable entre 10 a $40 \mathrm{mg} / \mathrm{ml}$ en asociación o no con lidocaína y puede realizarse cada 15 días dependiendo de la evolución $(23,24)$. Su principal desventaja radica en el dolor causado por la misma inyección. Una vez que la cicatriz se aplana debe detenerse el uso -incluso si la cicatriz sigue roja- ya que la piel podría ulcerarse. Otros efectos adversos incluyen la atrofia de la piel, la aparición de telangiectasias y la despigmentación (24).

\section{d) Láser}

Es un aparato que amplifica la luz por emisión estimulada de radiaciones para finalmente proyectar una luz monocromática (25). Existen diferentes tipos de acuerdo a la longitud de onda, pero los más utilizados para tratar cicatrices hipertróficas son el láser de colorante pulsado de $585 \mathrm{~nm}$ y el láser $\mathrm{Nd}$ :YAG de $1064 \mathrm{~nm}(26,27)$. Estos láseres vaporizan los vasos sanguíneos que llegan a la cicatriz, de manera que se impide la liberación local de las citoquinas inflamatorias, con ello, se limita el desarrollo de cicatrices patológicas $(28,29)$. El requerimiento energético para tratar las cicatrices hipertróficas es variable, así como el número de sesiones de tratamiento $(30,31)$. Los posibles efectos secundarios incluyen: la hiperpigmentación, la hipopigmentación, la formación de ampollas y la púrpura $(30,31)$.

Un metaanálisis demostró una tasa promedio sin recurrencia o de mejora del $72 \%$ con el láser de colorante pulsado PDL para el tratamiento de las cicatrices hipertróficas o queloides (10).

\section{e) Flavonoides}

Son sustancias extraídas de diversas plantas y dentro de sus bondades se usan para prevenir la formación de cicatrices patológicas. Diversos estudios han demostrado la eficacia de los geles de flavonoides, aunque los resultados son controversiales $(32,33)$. Por ejemplo, la quercetina -flavonoide encontrado en altas cantidades en la cebolla- demostró ser un agente anti fibrótico (34); este sería el mecanismo de acción para prevenir la formación de una cicatriz hipertrófica. Para tal fin, se recomienda su uso desde la segunda semana poscirugía por 2 veces al día hasta 6 meses (35).

\section{f) Toxina botulínica tipo A}

Es una neurotoxina derivada de la bacteria Clostridium botulinum que bloquea temporalmente la transmisión neuromuscular. Los estudios indican que 
esta denervación química temporal puede minimizar la formación de cicatrices hipertróficas al reducir el tono muscular local durante la cicatrización de la herida, pero también inhibiendo el ciclo celular del fibroblasto y por ende la síntesis de colágeno $(36,37)$.

La inyección intralesional es el método de administración preferido a dosis de 70-140 U por sesión mensual y hasta 3 sesiones $(38,39)$. Las inyecciones de toxina botulínica en el músculo orbicular de los labios inmediatamente después del cierre de la herida en adultos sometidos a una revisión de la cicatriz de labio mostraron resultados prometedores al disminuir la frecuencia y la severidad de las cicatrices hipertróficas (12). Asimismo, las investigaciones también reportan mejoría del dolor, la sensibilidad y el prurito en la cicatriz (38). Un metaanálisis demostró que la inyección intralesional de toxina botulínica tipo A fue más efectiva para inhibir la cicatriz hipertrófica que la inyección intralesional de corticosteroides o placebo; sin embargo, esta investigación no fue específica para las cicatrices hipertróficas posqueiloplastía (40).

\section{Tratamiento quirúrgico}

Ante una cicatriz hipertrófica posqueiloplastía que no mejora en el tiempo, se debe tener en cuenta la revisión quirúrgica. Sin embargo, antes de tomar esta decisión se debe analizar cuidadosamente las características de la cicatriz -morfología, madurez, distorsión de los tejidos- y añadir adecuadamente algún tratamiento conservador. La cirugía debe aplazarse hasta que madure la cicatriz o sea 12 meses, pues la cicatriz inmadura tiene un tejido bien vascularizado, edematoso y poco móvil, lo que hace difícil la revisión quirúrgica y aumenta el riesgo de complicaciones. Hasta que madure la cicatriz y para maximizar el resultado de una revisión se debe ofrecer algún tratamiento conservador (41).

La revisión quirúrgica comprende la exéresis de la cicatriz hipertrófica, así como la liberación de la tensión adyacente a la herida. Definitivamente el borde de la herida afrontada sin tensión cura con una cicatriz mínima, esto se logra mediante la aproximación del tejido profundo con puntos subdérmicos. El cierre de la piel del labio se debe realizar con puntos interrumpidos y la extracción de las suturas hasta 5 días después de la colocación. Las suturas permanentes como el polipropileno o el nylon generan menor reacción inflamatoria que las suturas absorbibles.
En niños, se combina de manera preventiva el uso del gel de silicona durante el día y la lámina de silicona por la noche (19). Cabe mencionar que las inyecciones de corticoesteroide en combinación con la cirugía mejoran las tasas de respuesta hasta en $95 \%$ y sin recurrencia de cicatrización patológica (42).

Es preciso reiterar que la opción quirúrgica debe evaluarse cuidadosamente, puesto que la cirugía induce a la inflamación, y por ende puede causar la recurrencia de la cicatriz hipertrófica (43). Indudablemente, la cicatriz hipertrófica posqueiloplastía debe operarse en caso se presenten áreas infectadas -como los quistes de inclusión- o cuando se produce la retracción del labio superior (41).

La cirugía de revisión debe basarse en los mismos principios descritos para la prevención de cicatrices patológicas: la técnica quirúrgica atraumática, el cierre sin tensión y la eversión adecuada de la piel. Asimismo, los esfuerzos para prevenir la infección de la herida y la administración de antibióticos perioperatorios deben ser una prioridad. Otro aspecto fundamental en esta cirugía, es la restauración de puntos de referencia anatómicos como el borde del bermellón del labio y la base del ala nasal, los cuales deben tener prioridad sobre la minimización de las características de la cicatriz (41). Al realizar la resección de la cicatriz hipertrófica, se debe evitar dejar un labio más largo (44).

\section{TERAPIA FUTURA}

La terapia futura o emergente se basa en la administración local de fármacos antitumorales e inmunomoduladores. La evidencia preliminar sugiere que la terapia con células madre mesenquimales $(45,46)$, la aplicación del injerto de grasa autóloga (47) y la avotermina (48), tienen potencial para el tratamiento contra la cicatrización excesiva. Sin embargo, estas nuevas terapias requieren más estudio antes de ser introducidas en la práctica clínica diaria (4).

\section{CONCLUSIONES}

Actualmente existen varias estrategias terapéuticas para el manejo de la cicatriz hipertrófica posqueiloplastía, pero ninguna es completamente satisfactoria. La evidencia reciente sugiere que la lámina o gel de silicona es la mejor opción para la prevención y el manejo inicial. Sin embargo, 
para aquellas cicatrices con respuesta baja o nula al tratamiento, hay diversas opciones que incluso pueden usarse en combinación, como las inyección intralesional de corticoides o de toxina botulínica, el láser y hasta la revisión quirúrgica. Si bien, hay una escasez de evidencia de nivel 1 con relación al manejo de la cicatriz hipertrófica posqueiloplastía, se sabe que el manejo temprano de la misma incidirá en una mejor apariencia estética y requerirá de menos tratamientos. Finalmente, se necesitan más estudios a gran escala, controlados y de alta calidad para determinar los protocolos de tratamiento más efectivos en todos los grupos étnicos.

\section{Declaración de conflictos de interés:}

El autor declara no tener conflictos de interés.

\section{Correspondencia:}

Alberto Córdova-Aguilar

Correo electrónico: alberto.cordova@upch.pe

\section{REFERENCIAS BIBLIOGRÁFICAS}

1. Dixon MJ, Marazita M, Beaty TH, Murray JC. Cleft lip and palate: understanding genetic and environmental influences. Nat Rev Genet. 2011; 12(3):167-78.

2. Soltani AM, Francis CS, Motamed A, Karatsonyi AL, Hammoudeh JA, Sanchez-Lara PA, et al. Hypertrophic scarring in cleft lip repair: a comparison of incidence among ethnic groups. Clin Epidemiol. 2012; 4:18791.

3. Shridharani SM, Magarakis M, Manson PN, Singh NK, Basdag B, Rosson GD. The emerging role of antineoplastic agents in the treatment of keloids and hypertrophic scars. Ann Plast Surg. 2010; 64(3):35561.

4. Ho JL, Yong JJ. Recent understandings of biology, prophylaxis and treatment strategies for hypertrophic scars and keloids. Int J Mol Sci. 2018; 19(3):711.

5. Gurtner GC, Werner S, Barrandon Y, Longaker MT. Wound Repair and Regeneration. Nature. 2008; 453:314-321.

6. Murawala P, Tanaka EM, Currie JD. Regeneration: The ultimate example of wound healing. Semin Cell Dev Biol. 2012;23(9):954-62.

7. Occleston NL, Metcalfe AD, Boanas A, et al. Therapeutic improvement of scarring: mechanisms of scarless and scar-forming healing and approaches to the discovery of new treatments. Dermatol Res Pract. 2010; 2010:405262. doi: 10.1155/2010/405262

8. Gallant C, Olson M, Hart D. Molecular, histologic, and gross phenotype of skin wound healing in red Duroc pigs reveals an abnormal healing phenotype of hypercontracted, hyperpigmented scarring. Wound Repair Regen. 2004; 12:305-19.

9. Slemp AE, Kirschner RE. Keloids and scars: a review of keloids and scars, their pathogenesis, risk factors, and management. Curr Opin Pediatr. 2006; 18(4):396402 .

10. Leventhal D, Furr M, Reiter D. Treatment of keloids and hypertrophic scars: a meta-analysis and review of the literature. Arch Facial Plast Surg. 2006; 8(6):3628.

11. Larrabee WFJ. Treatment of facial wounds with botulinum toxin A improves cosmetic outcomes in primates. Plast Reconst Surg. 2000; 105(6):1954-5.

12. Chang CS, Wallace CG, Hsiao YC, Chang CJ, Chen PK. Botulinum toxin to improve results in cleft lip repair. Plast Reconstr Surg. 2014; 134(3):511-6.

13. Scott JR, Muangman P, Gibran NS. Making sense of hypertrophic scar: a role for nerves. Wound Repair Regen. 2007; 15(suppl 1):S27-S31.

14. Wong VW, Akaishi S, Longaker MT, Gurtner GC. Pushing back: Wound mechanotransduction in repair. J Investig Dermatol. 2011; 131(11):2186-96.

15. Atkinson JA, McKenna, K, Barnett AG, McGrath DJ, Rudd M. A randomized, controlled trial to determine the efficacy of paper tape in preventing hypertrophic scar formation in surgical incisions that traverse Langer's skin tension lines. Plast Reconstr Surg. 2005; 116(6):1648-56.

16. Li-Tsang CW, Lau JC, Choi J, Chan CC, Jianan L. A prospective randomized clinical trial to investigate the effect of silicone gel sheeting (Cica-Care) on post-traumatic hypertrophic scar among the Chinese population. Burns. 2006; 32(6):678-83.

17. Majan JI. Evaluation of a self-adherent soft silicone dressing for the treatment of hypertrophic postoperative scars. J Wound Care. 2006; 15(5):1936.

18. Reish RG, Eriksson E. Scar treatments: Preclinical and clinical studies. J Am Coll Surg. 2008; 206(4):71930.

19. Chang CS, Wallace CG, Hsiao YC, et al. Clinical evaluation of silicone gel in the treatment of cleft lip scars. Sci Rep. 2018; 8(1):7422.

20. Mustoe TA. Evolution of silicone therapy and mechanism of action in scar management. Aesthetic Plast Surg. 2008; 32(1):82-92.

21. Roques C, Teot L. The use of corticosteroids to treat keloids: a review. Int J Low Extrem Wounds. 2008; 7(3):137-45.

22. Goutos I, Ogawa R. Steroid tape: A promising adjunct to scar management. Scars Burn Heal. 2017; 3:1-9.

23. Wong TS, Li JZ, Chen S, Chan JY, Gao W. The Efficacy of Triamcinolone Acetonide in Keloid Treatment: A Systematic Review and Meta-analysis. 
Front Med (Lausanne). 2016; 27(3):71.

24. Koc E, Arca E, Surucu B, Kurumlu Z. An open, randomized, controlled, comparative study of the combined effect of intralesional triamcinolone acetonide and onion extract gel and intralesional triamcinolone acetonide alone in the treatment of hypertrophic scars and keloids. Dermatol Surg. 2008; 34(11):1507-14.

25. Mathur RK, Sahu K, Saraf S, Patheja P, Khan F, Gupta PK. Low-level laser therapy as an adjunct to conventional therapy in the treatment of diabetic foot ulcers. Lasers Med Sci. 2017; 32(2):275-82.

26. Alster TS, Handrick C. Laser treatment of hypertrophic scars, keloids, and striae. Semin Cutan Med Surg. 2000; 19(4):287-92.

27. Koike S, Akaishi S, Nagashima Y, Dohi T, Hyakusoku H, Ogawa R. Nd:YAG Laser Treatment for Keloids and Hypertrophic Scars: An Analysis of 102 Cases. Plast Reconstr Surg Glob Open. 2014; 2(12): e272.

28. Alster T. Laser scar revision: Comparison study of 585-nm pulsed dye laser with and without intralesional corticosteroids. Dermatol Surg. 2003; 29(1):25-9.

29. Chan HH, Wong DS, Ho WS, Lam LK, Wei W. The use of pulsed dye laser for the prevention and treatment of hypertrophic scars in Chinese persons. Dermatol Surg. 2004; 30(7):987-94.

30. Tanzi EL, Alster TS. Laser treatment of scars. Skin Therapy Lett. 2004; 9(1): 4-7.

31. Akaishi S, Koike S, Dohi T, Kobe K, Hyakusoku H, Ogawa R. Nd:YAG Laser Treatment of Keloids and Hypertrophic Scars. Eplasty. 2012; 12:e1.

32. Beuth J, Hunzelmann N, van Leendert R, Basten R, Noehle M, Schneider B. Safety and efficacy of local administration of contractubex to hypertrophic scars in comparison to corticosteroid treatment. Results of a multicenter, comparative epidemiological cohort study in Germany. In Vivo. 2006; 20(2):27783.

33. Chung VQ, Kelley L, Marra D, Jiang SB. Onion extract gel versus petrolatum emollient on new surgical scars: Prospective double-blinded study. Dermatol Surg. 2006; 32(2):193-7.

34. Phan TT, Lim IJ, Sun L, et al. Quercetin inhibits fibronectin production by keloid-derived fibroblasts. Implication for the treatment of excessive scars. J Dermatol Sci. 2003; 33(3):192-4.

35. Ho WS, Ying SY, Chan PC, Chan HH. Use of onion extract, heparin, allantoin gel in prevention of scarring in Chinese patients having laser removal of tattoos: A prospective randomized controlled trial. Dermatol Surg. 2006; 32(2):193-7.

36. Xiao Z, Zhang M, Liu Y, Ren L. Botulinum toxin type a inhibits connective tissue growth factor expression in fibroblasts derived from hypertrophic scar. Aesthetic Plast Surg. 2011; 35(5):802-7.

37. Xiao Z, Zhang F, Lin W, Zhang M, Liu Y. Effect of botulinum toxin type A on transforming growth factor B1 in fibroblasts derived from hypertrophic scar: A preliminary report. Aesthetic Plast Surg. 2010; 34(4):424-7.

38. Elhefnawy AM. Assessment of intralesional injection of botulinum toxin type A injection for hypertrophic scars. Indian J Dermatol Venereol Leprol. 2016; 82(3):279-83

39. Shaarawy E, Hegazy RA, Abdel Hay RM. Intralesional botulinum toxin type A equally effective and better tolerated than intralesional steroid in the treatment of keloids: A randomized controlled trial. J Cosmet Dermatol. 2015; 14(2):161-6.

40. Bi M, Sun P, Li D, Dong Z, Chen Z. Intralesional Injection of Botulinum Toxin Type A Compared with Intralesional Injection of Corticosteroid for the Treatment of Hypertrophic Scar and Keloid: A Systematic Review and Meta-Analysis. Med Sci Monit. 2019; 22(25):2950-8.

41. Hu MS, Zielins ER, Longaker MT, Lorenz HP. Scar prevention, treatment, and revision. In: Gurtner GC, editor. Plastic Surgery: Principles. 4th ed. London: ELSEVIER; 2018. p. 196-212.

42. Chowdri NA, Masarat M, Mattoo A, Darzi MA. Keloids and hypertrophic scars: results with intraoperative and serial postoperative corticosteroid injection therapy. Aust N Z J Surg. 1999; 69(9):6559.

43. Mustoe TA, Cooter RD, Gold MH, et al. International Advisory Panel on Scar Management. International clinical recommendations on scar management. Plast Reconstr Surg. 2002; 110(2):560-71.

44. Rossell-Perry P. Malos resultados y complicaciones en la cirugía de fisura labio palatina. en: Gómez ERB, editor. Lima: Universal Nacional Mayor de San Marcos; 2018.

45. Lam MT, Nauta A, Meyer NP, Wu JC, Longaker MT. Effective delivery of stem cells using an extracellular matrix patch results in increased cell survival and proliferation and reduced scarring in skin wound healing. Tissue Eng Part A. 2013; 19(5-6):738-47.

46. Zonari A, Martins TM, Paula AC, et al. Polyhydroxybutyrate-co-hydroxyvalerate structures loaded with adipose stem cells promote skin healing with reduced scarring. Acta Biomater. 2015; 17:17081.

47. Negenborn VL, Groen JW, Smit J, Niessen FB, Mullender MG. The Use of Autologous Fat Grafting for Treatment of Scar Tissue and ScarRelated Conditions: A Systematic Review. Plast Reconstr Surg. 2016; 137(1):31e-43e.

48. So K, McGrouther DA, Bush JA, et al. Avotermin for scar improvement following scar revision surgery: a randomized, double-blind, within-patient, placebocontrolled, phase II clinical trial. Plast Reconstr Surg. 2011; 128(1):163-72. 